\title{
An outbreak of Middle East Respiratory Syndrome (MERS) due to coronavirus in Al-Ahssa Region, Saudi Arabia, 2015
}

H.E. El Bushra, ${ }^{1}$ M.N. Abdalla, ${ }^{1}$ H. Al Arbash, ${ }^{1}$ Z. Alshayeb, ${ }^{1}$ S. Al-Ali, ${ }^{2}$ Z. Al-Abdel Latif, ${ }^{2}$ H Al-Bahkit, ${ }^{2}$ O. Abdalla ${ }^{4}$, M. Mohammed ${ }^{4}$, H. Al-Abdely', M. Chahed, ${ }^{3}$ A.L. Lohiniva ${ }^{3}$, and A. Bin Saeed ${ }^{4}$

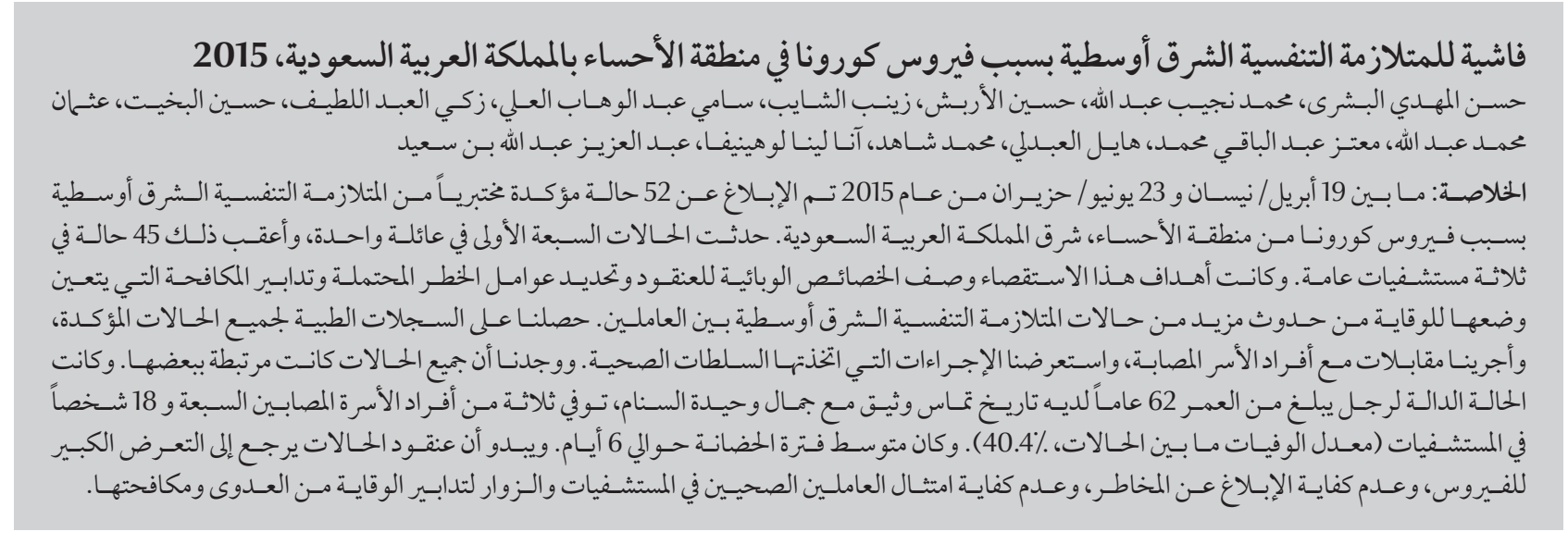

ABSTRACT Between 19 April and 23 June 2015, 52 laboratory-confirmed cases of Middle East Respiratory Syndrome due to coronavirus (MERS) were reported from Al-Ahssa region, eastern Saudi Arabia. The first seven cases occurred in one family; these were followed by 45 cases in three public hospitals. The objectives of this investigation were to describe the epidemiological characteristic of the cluster and identify potential risk factors and control measures to be instituted to prevent further occurrence of MERS. We obtained the medical records of all confirmed cases, interviewed the members of the affected household and reviewed the actions taken by the health authorities. All the cases were connected. The index case was a 62-year-old man with a history of close contact with dromedary camels; three of the seven infected family members and 18 people in hospitals died (case-fatality rate, 40.4\%). The median incubation period was about 6 days. The cluster of cases appeared to be due to high exposure to MERS, delayed diagnosis, inadequate risk communication and inadequate compliance of hospital health workers and visitors with infection prevention and control measures.

Arabie saoudite : flambée de syndrome respiratoire du Moyen-Orient (MERS) causé par le coronavirus dans la région d'Al-Ahssa en 2015

Entre le 19 avril et le 23 juin 2015, 52 cas confirmés en laboratoire de syndrome respiratoire du MoyenOrient (MERS) causé par le coronavirus ont été notifiés dans la région d'Al-Ahssa, partie orientale de I'Arabie saoudite. Les sept premiers cas sont survenus dans une seule famille; ils ont été suivis de 45 cas déclarés dans trois hôpitaux publics. Cette investigation avait pour objectifs de détailler les caractéristiques épidémiologiques de ce groupe de cas et d'identifier les facteurs de risque potentiels ainsi que les mesures de lutte à mettre en place afin d'empêcher la survenue de nouveaux cas de MERS. Nous avons consulté les dossiers médicaux de l'ensemble des cas confirmés, avons interrogé les membres des foyers touchés et passé en revue les interventions entreprises par les autorités sanitaires. Tous les cas étaient reliés entre eux. Le cas indicateur était un homme de 62 ans ayant eu des contacts étroits avec des dromadaires; trois des sept membres infectés de la famille et 18 patients hospitalisés sont décédés (taux de létalité : 40,4\%). La période d'incubation médiane était d’environ 6 jours. Le groupe de cas était vraisemblablement dû à une forte exposition au MERS, associée à un diagnostic tardif, une communication sur les risques inappropriée et une mauvaise observance des mesures de prévention et de lutte contre les infections par les personnels de santé de l’hôpital et les visiteurs.

'Saudi Field Epidemiology Program, Ministry of Health, Riyadh, Al-Ahssa, Saudi Arabia (Correspondence to: H.E. El Bushra: bestregards.h@gmail. com. ${ }^{2}$ Al-Ahssa Health Directorate, Saudi Arabia. ${ }^{3}$ WHO Country Office, Riyadh, Saudi Arabia. ${ }^{4}$ Deputyship for Public Health, Ministry of Health, Riyadh, Saudi Arabia

Received: 29/05/16; accepted 22/09/16 


\section{Introduction}

Middle East Respiratory Syndrome (MERS) is a viral respiratory disease associated with high case fatality rate (1). Primary human infections have been linked to direct or indirect exposure to dromedary camels infected with MERS coronavirus (MERS-CoV) in Saudi Arabia, Qatar and UAE (2-7). More than $85 \%$ of cases of MERS have been reported from Saudi Arabia (8). Major outbreaks of MERS occurred in health care facilities ranging from asymptomatic or mild illnesses to severe acute respiratory disease and death; the severe presentations of the disease occur among the elderly, the immunocompromised, and people with chronic underlying illnesses and are associated with a high fatality rate (9-12). To-date, no sustained human-to-human transmission has been documented (8).

On 20 April 2015, a case of MERS was reported in Al-Ahssa of eastern Saudi Arabia, followed by six confirmed cases in the same family of the index case. By 23 June 2015, a total of 52 laboratory-confirmed cases had been reported in the region, 45 secondary cases being reported in three public hospitals; and eight asymptomatic infections were found among health care workers.

Ministry of Health (MoH), Saudi Arabia deployed a joint mission with World Health Organization (WHO) to the region to investigate the outbreak to describe the epidemiological characteristics of the outbreak, to identify potential risk factors for the occurrence of the familial cluster of cases and the secondary cases in health care facilities and to identify the control measures to be instituted to prevent further nosocomial infections, including risk and outbreak communication.

\section{Material and methods}

\section{Background}

Al-Ahssa is the largest province in Saudi Arabia, covering $534000 \mathrm{~km}^{2}$, with a population of 1063112 in 2010. It has a dry, tropical climate, with a 5-month summer and a relatively cold winter. In addition to agricultural activities, there is intensive livestock-raising, making AlAhssa one of the major food producing areas of Saudi Arabia. There are three public hospitals in Al-Ahssa: Hospital A, B and C.

\section{Sources of data}

A complete list of all laboratory-confirmed cases of MERs was obtained from the Department of Preventive Medicine, Al-Ahssa General Directorate for Health Affairs. MERs diagnosis was conducted using Real-time reverse-transcription Polymerase Chain Reaction (rRT-PCR) assays for testing nasopharyngeal swabs and other lower respiratory specimens (13). Additional data were extracted from case investigation forms completed by field epidemiologists in Al-Ahssa region, by reviewing the medical records of the cases, by examining the log of messages and correspondence on "WhatsApp" among the health authorities of the region, the surveillance officers and MERs coordinators at the Ministry of Health in Riyadh.

The outbreak investigation team visited the family of the index case twice to obtain a full chronology of events and the relationships between the father (the index case) and other family members. All family members were interviewed in-depth to identify potential risk factors for acquiring MERS and nasopharyngeal swabs were collected for rRT-PCR testing. Serological tests for MERS-CoV were not done for logistical reasons.

\section{Infection and prevention control in Al-Ahssa hospitals}

Hospital A has 105 beds and serves a population of $300000-400000$ within its catchment area; it receives an average of 350 outpatients and 500 emergency room patients daily. Hospital B is a 73bed specialized tertiary hospital that serves the whole Al-Ahssa region and receives some patients from other regions. Hospital $\mathrm{C}$ receives on average about $700-800$ patients per day. The three hospitals have small dedicated units for infection prevention and control (IPC); each unit is composed from one to two doctors and a few nurses.

The directors of all three hospitals were asked to provide extensive information on implementation of recommended IPC measures, including detailed descriptions of all actions taken to contain the current outbreak of MERS and prevent secondary cases, with exact dates; a list of health care workers who were screened for MERS, their work stations in the hospitals, reason for testing, date of testing, date of reception of laboratory results and whether those who tested positive were symptomatic or not; dates of enforcement of use of personal protective equipment, triaging and other relevant IPC measures; a list of all the health workers involved in managing each case and whether they had used personal protection during the contacts; and a list of difficulties in fully implementing IPC measures. At Hospital C, we interviewed the nurses at the triage desk.

\section{Results}

The outbreak started as a cluster of seven cases in a family composed of 26 members living in a three-story building ( secondary attack rate $=23 \%)$. An additional 37 symptomatic and 8 asymptomatic confirmed cases occurred among health workers at Hospital B. The total number of symptomatic and asymptomatic cases was thus 52 . Three of the 
seven family members (the father, eldest son and daughter) died, as did 18 of the remaining symptomatic confirmed cases, for a case-fatality rate of $40.4 \%$. Table 1 summarizes the demographic characteristics of all cases.

\section{The index case and his family}

The index case was a 62-year-old man. His family owned a farm far from the house, where there were two young dromedary camels, both of which had ulcers. The father was reported to have close contact with camels, both on the farm and at regular camel markets. He used to spend the whole day in the farm with camels. The Ministry of Agriculture tested the two camels for MERS-CoV and found them to be IgG positive and IgM negative.

The 26 family members lived in a crowded, poorly ventilated house. The mother had diabetes, hypertension and renal problems, and the father and the eldest son had smoked shisha for many years. The family was not well educated, and the local health authority assessed the general level of hygiene as very low. They had very limited contact with their neighbours. They reported that they were aware of the public health messages about MERS. Although the father was eventually hospitalized, his son did not inform other family members of the diagnosis, even after he himself contracted the disease. After his brothers and sisters started showing symptoms of the disease, the youngest brother also refused to be admitted to hospital.

\section{Cases of MERS at hospitals}

Six confirmed cases of MERs were diagnosed at Hospital A after admission of members of the family: three doctors and three inpatients. The first physician accompanied a patient from the Hospital A to the Hospital B. The second case occurred in a doctor in the intensive care unit who intubated a critically ill patient without putting on personal protective equipment; he was still asymptomatic on the day of the visit. The third physician ran an orthopedic trauma clinic at the Hospital; he denied contact with suspected or confirmed cases of MERS and believed that he had been infected by his wife, who worked at the Hospital B; however, a nasopharyngeal swab taken from his wife was negative for MERS-CoV.

Fifteen confirmed cases of MERS were referred from Hospital A to Hospital B, and were later referred to Hospital C. Of the referred cases, most patients had spent at least 2 days at Hospital A before being referred to Hospital $\mathrm{B}$. The administration of Hospital B believed that some of the cases contracted the infection before referral to Hospital; and symptoms of MERS started to appear after their admission. A staff physiotherapist at Hospital B contracted MERS from a patient in the outpatient department and was severely ill for 2 days. He was nursed at home by his parents; a father who was immune-compromised (had a lung transplant) and his old mother. Neither the father nor the mother became infected with MERS. Health care workers in Hospitals A and B, who were exposed to MERS cases, were screened for MERS-CoV.

\section{The epidemic curve}

An epidemic curve was plotted according to the date of onset of symptoms (Figure 1). The curve shows two

\begin{tabular}{lc}
\hline Table 1 Numbers of cases of Middle East Respiratory Syndrome due to coronavirus (MERS), Al-Ahssa, Saudi Arabia, April-June \\
2015 & No. of cases (\%) \\
\hline Total & 52 \\
Males & $31(59.6 \%)$ \\
Secondary cases & 51 \\
Case-fatality rate & $21(40.4 \%)$ \\
Family & 7 \\
Secondary attack rate in family & $6(23.1 \%)$ \\
Symptomatic cases & $44(84.6 \%)$ \\
Asymptomatic infections & $8(15.4 \%)$ \\
Non-health workers & $36(69.2 \%)$ \\
All health workers & $16(30.8 \%)$ \\
Male health workers & $9(17.3 \%)$ \\
Female health workers & $7(13.5 \%)$ \\
Medical doctors (senior medical officer, two residents, orthopedic surgeon, anesthesiologist) & $6(37.5 \%)$ \\
Nurses & $8(50 \%)$ \\
Physiotherapist & $1(6.3 \%)$ \\
Security staff * & $1(6.3 \%)$
\end{tabular}

*The security staff at the health facilities deals directly with patients and is considered as a paramedical health care worker. 
distinct clusters of cases; the first comprised the seven cases among members of the family, and the second consists of secondary cases associated with visiting, admission to, or working at the three public hospitals. All confirmed cases of MERS were admitted to Hospital C.
Figure 1 shows that the cluster of cases in the affected family preceded those in the three public hospitals. The median incubation period was about 6 days. Subsequent cases were first diagnosed among patients and health workers at Hospital B. All cases were found to be connected. Some of the patients were already admitted at Hospital B before becoming infected.

The eldest brother started to have symptoms on 28 April, the onset of symptoms in five of the seven infected members of the family occurred within
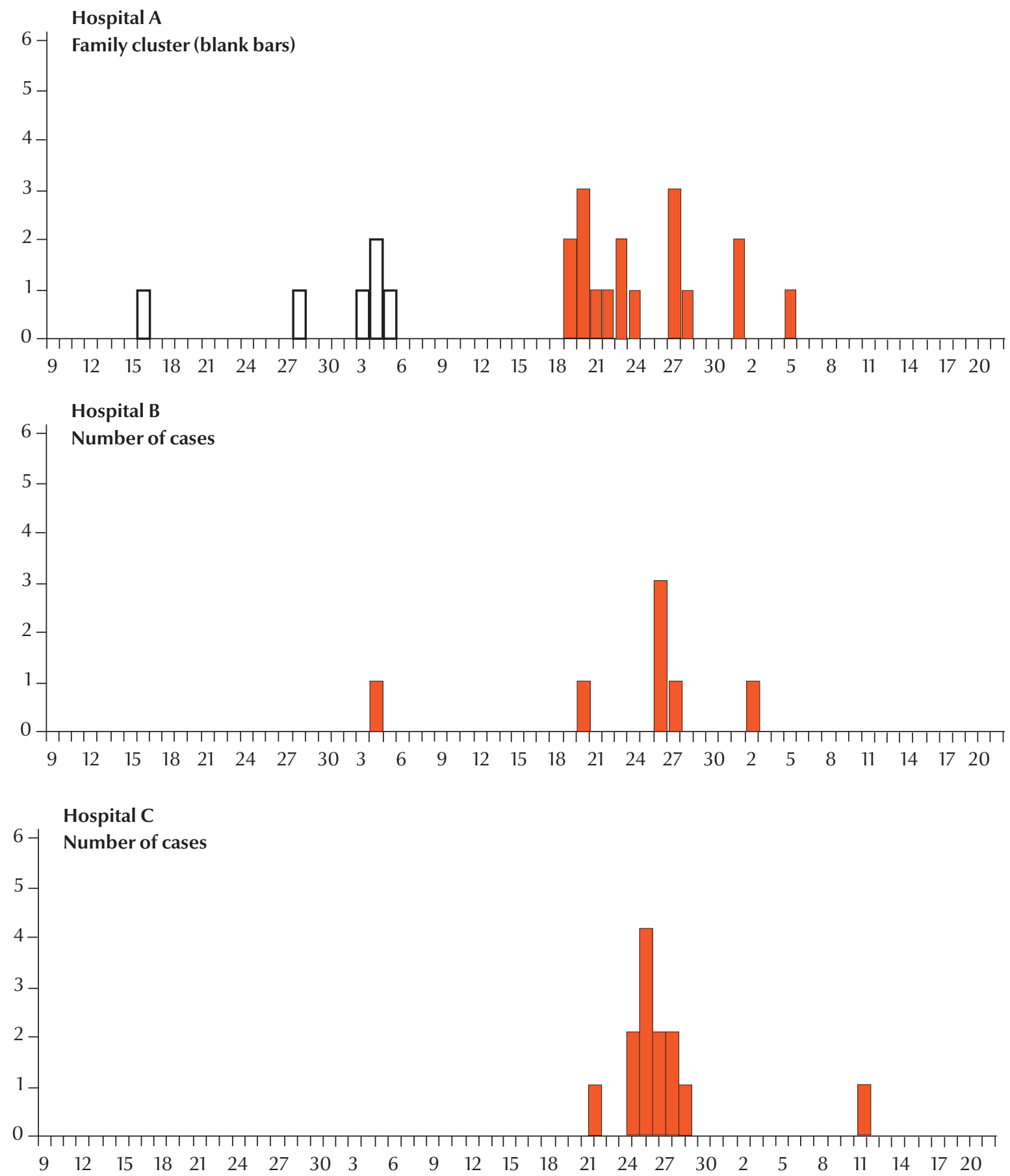

Date of onset of symptoms by international week

Figure 1. Epidemic curve of MERS showing distribution of cases of MERS by date of onset in Al-Ahssa, Saudi Arabia, 2015 
2 days (4-5 May); six of the confirmed cases had been in Hospital B for 6-20 days before the onset of symptoms; four other patients had visited Hospital $B$ for medical consultations or an invasive procedure few days before they experienced symptoms of the disease. Eight asymptomatic health workers in Hospital B were identified during the outbreak; none had had any direct contact with the family.

\section{Discussion}

The emergence of seven cases of MERS disease within one family and the rapid appearance of additional cases among health workers and patients in one of the hospitals raised serious concern among public health authorities, especially as the outbreak was associated with a relatively high case-fatality rate and occurred after a long period with no MERS cases. It is generally considered that transmission is mediated primarily by mutations with increased affinity to human receptors (14); alternatively, it has been proposed a "super-spreader" has emerged (15). However; in-depth interviews with some of the family members, indicated that the probable risk factors were exposure of the index case to a high dose of corona virus and delayed diagnosis due to denial by family members; these factors were exacerbated by improper risk communication and inadequate compliance with IPC measures in the hospitals.

The appearance of symptoms in five of the seven infected family members within 2 days indicated that they were likely infected by the eldest brother. The occurrence of large familial clusters of severe cases of MERS is relatively uncommon (16-18). The secondaryattack rate in the family was much higher (23\%) than those reported in previous studies (about 5\%) (19-21). Factors that probably contributed to occurrence of many cases in the family were probably related to the crowded living conditions, poor hygiene and close relationships among the male family members. The family's behaviour indicates the importance of training of health workers to ensure that patients fully understand the importance of adherence and compliance with public health messages. Psychosocial counselling and support should be available to affected families, and strategies should be devised to ensure appropriate outbreak communication, via the best channels, to ensure visibility and to target the primary audiences.

No new cases of MERS appeared after the identification of asymptomatic infected health workers, indicating that asymptomaticinfections in health workers could play a role in transmission of MERS within health care facilities (HCFs) (22). The use of different case definitions for MERs by different hospitals may partially explain why some HCFs reported more suspected cases. A recent unpublished study (Al-Zahrani, Ministry of Health) found that the positive predictive value of the current case definition is $0-4 \%$. Many recent studies indicate that fever is only irregularly associated with the onset of MERS (9, 12). Delays in diagnosis of MERS were due partially to repeated negative or inconclusive nasopharyngeal swabs, with confirmed positive results only after a third or fourth specimen (23). In one instance, confirmation was obtained after burial of a deceased patient. False negative laboratory results has been attributed to timing, quality, technique of specimen collection and transportation to a reference laboratory as well as variability in virus shedding among patients (11).

The interventions for preventing primary and secondary cases are different. Increasing public awareness through risk communication is the core activity for reducing or preventing the emergence of primary cases; whereas efficient IPC programmes are required to prevent secondary infections $(9,12,19,24,25)$. The occurrence of secondary cases of MERS in hospitals is an indicator of laxity and complacency in implementing IPC measures or shortage of human resources, equipment, supplies or supervision. The current outbreak showed that there are still many gaps in the IPC programmes in hospitals in Al-Ahssa region, despite an overall improvement in Saudi Arabia. The directors of hospitals and health services require guidance and training to improve IPC in their HCFs. A module should be provided on additional IPC measures to be observed during response to and management of outbreaks of infectious respiratory infections such as MERS.

MERS is a droplet infection (26, 27); however, interviews with doctors showed that many were poorly informed about MERS-CoV, particularly the mode of transmission of the virus, many were believing that it is an airborne disease. The major challenge in implementing IPC programmes is to change the behaviour of health workers, such as ensuring hand hygiene; another challenge is maintaining good practice between outbreaks. There is a lack of clear leadership for IPC in the hospitals and no clear division of roles and responsibilities or guidance on dealing with health workers who repeatedly violate IPC directions. Some authorities have called for penalties or punishments, while others have called for clauses in public health law to limit the movement of suspected cases of MERS-CoV $(28,29)$.

Continuing education on IPC should be provided, although it should not give a false sense of security to hospital administrators. IPC must remain everybody's responsibility, with adequate stockpiles of equipment and supplies. Health care workers and HCFs should follow the auditing system for IPC practices developed by Ministry of Health, Saudi Arabia, in collaboration with Central Board for Accreditation of Healthcare Institutions (CBAHI). The IPC auditing system is designed 
to ensure sustained compliance to IPC guidelines using a scoring method; the audits monitor performance of $\mathrm{HCFs}$, and implementation of immediate corrective actions as deemed necessary (30). The routine IPC assessment tools need to be revised to ensure that it is also appropriate for use during outbreaks which would require additional IPC measures. There is need to address and monitor the behaviour and practices of health workers and hospital management system.

During hospital outbreaks many hospitals reduced admissions, stopped elective admissions and surgeries or closed certain units during disease outbreaks (31). While such actions would increase the bed capacity of that institution, some hospital administrators tend to take such measures to avoid functioning during outbreaks. Reduction of the workload during an outbreak could be considered an opportunity to identify weaknesses, train staff and improve the preparedness and readiness of hospitals for similar events in the future. Hospitals should document and share their experiences with MERs.

The outbreak made it clear that no hospital or health worker is immune against MERs. Hospital B has always considered itself a centre of excellence and was confident of the quality of the medical care it provides; however, referrals of patients from other hospitals rendered Hospital B susceptible to MERs. Emergence of MERs in Hospital B pushed its administration to monitor hand hygiene of its health workers through nursing stations; noncompliant health workers, including senior physicians, were reported to the hospital administration immediately.

Issuance of a clear statement by the Director-General of Al-Ahssa Health Directorate was needed to support strict implementation of the visitor policy. Other problems that compromise proper IPC include inadequate quantities of up-to-date equipment; hospital rooms, cupboards and corridors are full of obsolete equipment. Hospital A lacks a professional IPC officer and does not have an adequate number of dedicated IPC nurses.

The Scientific Advisory Council of the Ministry of Health has recommended that clinical triage be used for early identification of patients with acute respiratory illness in emergency rooms and clinics to prevent transmission of MERS and other respiratory viruses (32). The outbreak investigation team identified practical difficulties in visual and structured triaging of patients, including poor understanding of the triaging concept and logistics. Many hospitals in Al-Ahssa found it difficult to spare adequate space for proper triaging, such as special rooms for assessment and screening, isolation rooms and separate elevators for suspected MERS cases needed for proper implementation. The health workers assigned to triaging were not adequately trained and not fully dedicated to this activity. A detailed checklist of all requirements could be useful for establishing a functional triaging system at HCFs.

The possibility that referrals between hospitals contributed in spread of MERS from one hospital to another may not be the only factor. Some cases had been hospitalized at Hospital B for longer than the longest incubation period of MERS and must therefore have been infected during their hospitalization; however, some newly diagnosed cases that reported had visited the outpatient department at Hospital B might have been exposed to MERS corona virus at other hospitals. Referrals to Hospital B for further management of cardiac conditions might have been misdiagnosed or undiagnosed cases of MERS, as patients with cardiac disease and with MERS both present with cough and breathlessness. A system could be devised whereby each hospital receives alerts about newly diagnosed cases of MERS in other hospitals. This could be useful in reducing the risk of passing MERS infection from one hospital to another.

Outbreak communication is an integral component of the response to minimize panic and reduce the circulation of rumours. Health authorities should transparently announce the occurrence of an outbreak as soon as possible and address potential public concerns. Timely, appropriate outbreak communication will maintain the trust of the community and ensure their participation in control measures. The main aim of health risk communicators is to transmit information clearly to encourage behavioural changes to reduce risk. Compliance with health education messages depends on the extent to which they address public concerns.

During the outbreak, the DirectorGeneral for Health Affairs in Al-Ahssa decided that the IPC officer should report directly to his office, and the terms of reference of the IPC officer at the regional level were revised accordingly. This action probably increased access of additional resources for emerging public health crises and expedited the purchase of necessary equipment and supplies.

\section{Acknowledgements}

The outbreak investigation team acknowledges the contribution of the health authorities in Al-Ahssa region throughout the investigation, working as our partners and facilitating access to data and interviews. Special thanks go to the Director-General for Health Affairs and the Directorates for Public Health and IPC. We also thank the directors and IPC staff at the Hospital A, B and C for their openness and their hard work to stop the infection.

\section{Funding: None.}

Competing interests: None declared. 
1. Zumla A, Hui DS, Perlman S. Middle East respiratory syndrome. Lancet. 2015 Sep 5;386(9997):995-1007. PMID:26049252

2. Memish ZA, Cotten M, Meyer B, Watson SJ, Alsahafi AJ, Al Rabeeah AA, et al. Human infection with MERS coronavirus after exposure to infected camels, Saudi Arabia, 2013. Emerg Infect Dis. 2014 Jun;20(6):1012-5. PMID:24857749

3. Reusken CB, Farag EA, Jonges M, Godeke GJ, El-Sayed AM, Pas SD, et al. Middle East respiratory syndrome coronavirus (MERS-CoV) RNA and neutralising antibodies in milk collected according to local customs from dromedary camels, Qatar, April 2014. Euro Surveill. 201406 12;19(23):20829. PMID:24957745

4. Hemida MG, Perera RA, Al Jassim RA, Kayali G, Siu LY, Wang P, Chu KW, Perlman S, Ali MA, Alnaeem A, Guan Y. Seroepidemiology of Middle East Respiratory Syndrome (MERS) coronavirus in Saudi Arabia (1993) and Australia (2014) and characterisation of assay specificity. Euro surveillance: bulletin Europeen sur les maladies transmissibles= European communicable disease bulletin. $2013 \mathrm{Dec} ; 19(23)$.

5. Meyer B, Müller MA, Corman VM, Reusken CB, Ritz D, Godeke GJ, et al. Antibodies against MERS coronavirus in dromedary camels, United Arab Emirates, 2003 and 2013. Emerg Infect Dis. 2014 Apr;20(4):552-9. PMID:24655412

6. Azhar El, El-Kafrawy SA, Farraj SA, Hassan AM, Al-Saeed MS, Hashem AM, et al. Evidence for camel-to-human transmission of MERS coronavirus. N Engl J Med. 2014 Jun 26;370(26):2499505. PMID:24896817

7. Müller MA, Meyer B, Corman VM, Al-Masri M, Turkestani A, Ritz D, et al. Presence of Middle East respiratory syndrome coronavirus antibodies in Saudi Arabia: a nationwide, cross-sectional, serological study. Lancet Infect Dis. 2015 May;15(5):559-64. PMID:25863564

8. World Health Organization. Middle East Respiratory Syndrome coronavirus (MERS-CoV). Fact sheet $\mathrm{N}^{\circ} 401$ June 2015. (http://www.who.int/mediacentre/factsheets/mers-cov/ en/, accessed 16 March 2016).

9. Assiri A, McGeer A, Perl TM, Price CS, Al Rabeeah AA, Cummings DA, et al.; KSA MERS-CoV Investigation Team. Hospital outbreak of Middle East respiratory syndrome coronavirus. N Engl J Med. 2013 Aug 1;369(5):407-16. PMID:23782161

10. Balkhy $\mathrm{HH}$, Alenazi TH, Alshamrani MM, Baffoe-Bonnie $\mathrm{H}$, Arabi Y, Hijazi R, et al. Description of a Hospital Outbreak of Middle East Respiratory Syndrome in a Large Tertiary Care Hospital in Saudi Arabia. Infect Control Hosp Epidemiol. 2016 Jul 18;•••:1-9. PMID:27426423

11. 11. Arabi YM, Arifi AA, Balkhy HH, Najm H, Aldawood AS, Ghabashi A, et al. Clinical course and outcomes of critically ill patients with Middle East respiratory syndrome coronavirus infection. Ann Intern Med. 2014 Mar 18;160(6):389-97. PMID:24474051

12. Al-Tawfiq JA, Assiri A, Memish ZA. Middle East respiratory syndrome novel corona MERS-CoV infection. Epidemiology and outcome update. Saudi Med J. 2013 Oct;34(10):991-4. PMID:24145930

13. Guery B, Poissy J, el Mansouf L, Séjourné C, Ettahar N, Lemaire $X$, et al.; MERS-CoV study group. Clinical features and viral diagnosis of two cases of infection with Middle East Respiratory Syndrome coronavirus: a report of nosocomial transmission. Lancet. 2013 Jun 29;381(9885):2265-72. PMID:23727167

14. Lu G, Wang Q, Gao GF. Bat-to-human: spike features determining 'host jump' of coronaviruses SARS-CoV, MERSCoV, and beyond. Trends Microbiol. 2015 Aug;23(8):468-78. PMID:26206723
15. Kim SW, Yang TU, Jeong Y, Park JW, Lee KJ, Kim KM, et al. Middle East Respiratory Syndrome coronavirus outbreak in the Republic of Korea, 2015. Osong Public Health Res Perspect. 2016. Forthcoming

16. Health Protection Agency (HPA) UK Novel Coronavirus Investigation team. Evidence of person-to-person transmission within a family cluster of novel coronavirus infections, United Kingdom, February 2013. Euro surveillance: bulletin Européen sur les maladies transmissibles= European communicable disease bulletin. 2013 Mar 14;18(11):20427.

17. Memish ZA, Zumla Al, Al-Hakeem RF, Al-Rabeeah AA, Stephens GM. Family cluster of Middle East respiratory syndrome coronavirus infections. N Engl J Med. 2013 Jun 27;368(26):2487-94. PMID:23718156

18. Abroug F, Slim A, Ouanes-Besbes L, Hadj Kacem MA, Dachraoui F, Ouanes I, et al.; World Health Organization Global Outbreak Alert and Response Network Middle East Respiratory Syndrome Coronavirus International Investigation Team. Family cluster of Middle East respiratory syndrome coronavirus infections, Tunisia, 2013. Emerg Infect Dis. 2014 Sep;20(9):1527-30. PMID:25148113

19. Drosten C, Meyer B, Müller MA, Corman VM, Al-Masri M, Hossain R, et al. Transmission of MERS-coronavirus in household contacts. N Engl J Med. 2014 Aug 28;371(9):828-35. PMID:25162889

20. Al-Tawfiq JA, Memish ZA. Middle East respiratory syndrome coronavirus: epidemiology and disease control measures. Infect Drug Resist. 201411 03;7:281-7. PMID:25395865

21. Alagaili AN, Briese T, Mishra N, Kapoor V, Sameroff SC, Burbelo PD, et al. Middle East respiratory syndrome coronavirus infection in dromedary camels in Saudi Arabia. MBio. 201402 25;5(2):e00884-14. PMID:24570370

22. Memish ZA, Zumla Al, Assiri A. Middle East respiratory syndrome coronavirus infections in health care workers. N Engl J Med. 2013 Aug 29;369(9):884-6. PMID:23923992

23. Arabi YM, Arifi AA, Balkhy HH, Najm H, Aldawood AS, Ghabashi A, et al. Clinical course and outcomes of critically ill patients with Middle East respiratory syndrome coronavirus infection. Ann Intern Med. 2014 Mar 18;160(6):389-97. PMID:24474051

24. Omrani AS, Matin MA, Haddad Q, Al-Nakhli D, Memish ZA, Albarrak AM. A family cluster of Middle East Respiratory Syndrome Coronavirus infections related to a likely unrecognized asymptomatic or mild case. Int J Infect Dis. 2013 Sep;17(9):e668-72. PMID:23916548

25. World Health Organization. Infection prevention and control during health care for probable or confirmed cases of novel coronavirus (nCoV) infection. Interim guidance. Geneva: World Health Organization; 2015 (http://www.who.int/csr/ disease/coronavirus_infections/ipc-mers-cov/en/).

26. Chung SJ, Ling ML, Seto WH, Ang BS, Tambyah PA. Debate on MERS-CoV respiratory precautions: surgical mask or N95 respirators? Singapore Med J. 2014 Jun;55(6):294-7. PMID:25017402

27. Hui DS, Peiris M. Middle East Respiratory Syndrome. Am J RespirCritCareMed. 2015 Aug 1;192(3):278-9. PMID:26120749

28. Ministry of Health. MERS: legal issues. Weekly Monitor MERSCoV; 2: 27. (http://www.moh.gov.sa/en/CCC/Documents/ Volume-2-Issue-27-Tuesday-July-13-2016\%E2\%80\%8B.pdf).

29. Centers for Disease Control and Preventions (CDC). Specific Laws and Regulations Governing the Control of Communicable Diseases. (http://www.cdc.gov/quarantine/criteria-for- 
recommending-federal-travel- estrictions.html, accessed 17 August 2016).

30. Ministry of Health. Infection Prevention and Control (IPC) measures for MERS-CoV. Weekly Monitor MERS-CoV;1:9 (22 Dec 2015) (http://www.moh.gov.sa/en/CCC/Documents/ Volume-1-Issue-9-Tuesday-December-22-2015.pdf).

31. Balkhy $\mathrm{HH}$, Alenazi $\mathrm{TH}$, Alshamrani MM, Baffoe-Bonnie $\mathrm{H}, \mathrm{Al}-$ Abdely HM, El-Saed A, et al. Notes from the Field: Nosocomial Outbreak of Middle East Respiratory Syndrome in a Large Ter- tiary Care Hospital-Riyadh, Saudi Arabia, 2015. MMWR Morb Mortal Wkly Rep. 201602 19;65(6):163-4. PMID:26890816

32. Infection prevention/control and management guidelines for patients with Middle East Respiratory Syndrome coronavirus (MERS-CoV) infection. 2nd ed. Riyadh: Scientific Advisory Council, Ministry of Health; 2014. 